\title{
Optimization of I/O Intensive Genome Assemblies on the Cori Supercomputer with Burst Buffer
}

\author{
Joshua Pritchett \\ Joint Genome Institute \\ Lawrence Berkeley National Laboratory \\ jpritch@uab.edu
}

\author{
Bill Andreopoulos \\ Joint Genome Institute \\ Lawrence Berkeley National Laboratory \\ wandreopoulos@lbl.gov
}

\begin{abstract}
Since the development of next generation sequencing technologies, genome assembly has become one of the most computational and I/O intensive analyses done on the genomic data. The flood of genomic sequence data has increased the demand for more efficient genome assembly workflows. One of the assemblers being used for this purpose, Falcon, a long fragment sequence assembler, has several parts that are very I/O intensive as they read and write many files to disk. While the computationally expensive parts of assembly are more efficient with Falcon than other assemblers, the $\mathrm{I} / \mathrm{O}$ while reading and writing files to disk is a bottleneck. It has been observed that the wall clock runtime of the Falcon pipeline can be reduced by copying data files to local disk rather than rely on the Lustre parallel file system or NFS. Making a higher bandwidth available to the application should allow the application to read/write a large amount of data faster, such that I/O is not a bottleneck for genome assembly.

In this article, we investigate using the NERSC's Cori, Cray XC40 supercomputer, to improve the runtimes of genome assemblies at the Joint Genome Institute. We find that Cori gives a significant runtime improvement over the older highperformance computing cluster when running I/O intensive genome assemblies. This confirms the benefits of running assemblies on Cori. Using a local disk on a cluster node also results in a runtime improvement as expected. However, the Burst Buffer technology that is meant to act as local disk for Cori did not give a significant improvement. This was due to the nature of $\mathrm{I} / \mathrm{O}$ with Falcon that involves writing many small files rather than one very large file.
\end{abstract}

\section{Categories and Subject Descriptors}

C.4 [Computer Systems Organization]: Performance of Systems; C.5.1 [Computer System Implementation]: Large and Medium ("Mainframe") Computers-Super (very large) computers; J.3 [Computer Applications]: Life and Medical Sciences-Biology and Genetics

Permission to make digital or hard copies of all or part of this work for personal or classroom use is granted without fee provided that copies are not made or distributed for profit or commercial advantage and that copies bear this notice and the full citation on the first page. Copyrights for components of this work owned by others than ACM must be honored. Abstracting with credit is permitted. To copy otherwise, or republish, to post on servers or to redistribute to lists, requires prior specific permission and/or a fee. Request permissions from permissions@ acm.org.

$B C B^{\prime} 16$, October 2-5, 2016, Seattle, WA, USA.

Copyright 2016 ACM 978-1-4503-4225-4/16/10 ...\$15.00.

http://dx.doi.org/10.1145/2975167.2985685.

\section{General Terms}

Performance, Measurement

\section{Keywords}

Assembly, Genome, Optimization, Profiling, Burst Buffer, High Performance Computing, Supercomputer, Cori, Falcon, Cluster, Lustre, GPFS

\section{INTRODUCTION}

The introduction of genome sequencing technologies and increased computational resources together have revolutionized biology. Following the chain termination method of sequencing, developed in 1977, by Frederick Sanger, the first genomes of cellular organisms were sequenced, the bacterial species Haemophilus influenzae and Mycoplasma genitalium. By 2008, the genomes of more than 800 bacteria and 100 eukaryotes had been sequenced, including the genomes of several human individuals. Scientists are now sequencing microbial, plant and human genomes faster than ever before for applications that include precision medicine, disease susceptibility testing and alternative biofuels. As of 2010, the traditional GenBank divisions, central repository of genetic sequence information hosted by the NCBI, contain 106 billion nucleotide bases from 108 million individual sequences, with 11 million new sequences added since 2009 , with the number of sequence records doubling approximately every 35 months 3 . Modern sequencing instruments can read DNA fragments of up to 20,000 nucleotide bases (commonly just 600-10,000 nucleotide bases), which is orders of magnitude shorter than the genomes of most living organisms. For several years, many thought such limitations would restrain the sequencing of large genomes, such as complex bacteria, plants and humans. Today, however, the sequencing of bacteria (millions of nucleotide bases in length) is done routinely and the sequencing of 1000 human genomes ( 3 billion bp in length, each) is a reality. In part, these possibilities are due to advancement of new sequencing methods, such as Illumina and PacBio 9. The sequencing process involves shearing the genome of an organism into multiple small fragments, each of which is sequenced separately. Illumina technology can sequence fragments of up to 500 nucleotide bases, while PacBio technology can sequence up to 20,000 nucleotide base fragments. The resulting DNA fragments are combined into a reconstruction of the original genome using special computer programs called genome assemblers 14 . 
The Joint Genome Institute (JGI) performs sequencing on behalf of the U.S. Department of Energy Bioenergy Research Centers. The mission of the JGI is to advance genomics in support of clean energy generation and environmental characterization and cleanup. The genome assembly group at the JGI works on assembling genomes from microbes and fungi. The sequences are generated mainly by Illumina and PacBio technologies. After receiving the fragmented genomic datasets, the genome assembly group assembles the fragments to produce the entire genomic DNA sequence. The software used includes Spades [2], Velvet 15], or ALLPATHS 6] genome assemblers for short fragments, and the Falcon genome assembler for long fragments [11, 7]. The genome assemblies performed at JGI run on NERSC's Genepool high performance computing (HPC) cluster or the Cray XC40 supercomputer known as Cori. With demand for DNA sequencing increasing, the runtime and memory requirements for the genome assembly process are expected to increase over time. Execution runtime and memory usage should not be limiting factors in assembling genome sequences. While many genome assembly algorithms have been developed that are scalable and memory efficient, it is also important to understand how the computational resources used will affect the execution runtime, disk and memory usage and assembly quality 10].

Our goal in this study is to optimize the Falcon assembler that is used for assembling genomes from long fragment sequencing technologies. Similar to other long fragment genome assemblers, several parts of the Falcon assembly are very $\mathrm{I} / \mathrm{O}$ intensive as they read and write many large files $(>=100 \mathrm{~GB}$ of data) to disk per genome. While the computationally expensive parts of assembly are more efficient with Falcon than other assemblers, the I/O while reading and writing files to disk is a bottleneck. The JGI wants to move its genome assembly and other analysis pipelines to Cori in the years 2016-2018, given the rapidly increasing computational demands of the various scientific research programs. Cori is expected to provide a significant increase in computational capabilities. Cori will provide high bandwidth access to existing data stored by the various research projects. Additionally, Cori will be a first step in transitioning users to more energy efficient many-core architectures. For the purpose of transitioning to Cori, JGI needs to analyze the performance of its various pipelines on Cori to understand the gains and benefits that can be achieved.

In this paper we evaluated if the Cray XC40 supercomputer, also known as Cori, and the Burst Buffer technology can improve the performance of the Falcon genome assembler that is I/O intensive and writes hundreds of thousands of files to disk. In this study, we tried to use Cori and the Burst Buffer to increase the I/O bandwidth for Falcon assembly of microbial and fungal genomes from long fragments (PacBio).

This article is organized as follows. Section 2 describes the specifications of the Genepool, Cori and Burst Buffer. In Section 3 we describe the data sets used for benchmarking and their unique characteristics that make our runtime profiling study different from other studies. Section 4 explains how the results were evaluated and runtimes were compared, including our use of Docker. Section 5 describes the results in detail. Section 6 is a discussion of interesting observations related to adapting Cori for the JGI's bioinformatics pipelines. We conclude with our plans for future work.

\section{GENEPOOL, CORI AND BURST BUFFER SPECIFICATIONS}

The Genepool system is a computing cluster dedicated to the DOE JGI's computing needs. Genepool is made up of a heterogeneous collection of nodes to serve the diverse workload of the JGI users. While there are 212 nodes with 16 cores (Xeon E5-2670) and 120GB of RAM, there are only 2 nodes with 32 cores (Xeon E5-4650L) and 1TB of RAM. On the larger nodes it takes longer to schedule jobs via UGE than on the smaller nodes. Genepool uses a 2.7PB GPFS based file system, which is named projectb. Each Genepool node also has a local disc of size $1.8 \mathrm{~TB}-3.6 \mathrm{~TB}$, which is named local scratch.

Cori is NERSC's newest supercomputer system. Cori consists of 1,630 compute nodes with $128 \mathrm{~GB}$ of memory per node and two $2.3 \mathrm{GHz}$ 16-core Intel Haswell processors per node. Each core has its own L1 and L2 caches, with 64 $\mathrm{KB}$ (32 KB instruction cache, $32 \mathrm{~KB}$ data) and $256 \mathrm{~KB}$, respectively; there is also a 40MB shared L3 cache per socket. Cori uses the Cray-developed Aries high speed "dragonfly" topology interconnect, which is known to improve runtime and application scalability. Cray uses a Lustre file system with $30 \mathrm{~PB}$ of disk and $>700 \mathrm{~GB} /$ second I/O bandwidth, which is named cscratch. Unlike Genepool that uses UGE for scheduling jobs to run, Cori uses the SLURM workload manager, which is open-source and free and saves costs.

Cori is also designed to accelerate data-intensive applications through an installation of Cray's DataWarp (Burst Buffer), which provides high bandwidth and low latency I/O. The Burst Buffer on Cori is a layer of non-volatile storage that sits between the processors' memory and the parallel file system. The Burst Buffer is expected to accelerate I/O performance of application on Cori. Burst Buffers can be realized in a variety of ways. In the case of Cray's DataWarp implementation that is used at NERSC [8], it is achieved through SSD (solid-state drive) technologies in I/O nodes that are directly connected to the high-speed network, rather than in compute nodes. The flash memory for Cray DataWarp is attached to Burst Buffer nodes that are packaged two nodes to a blade. Each Burst Buffer node contains a Xeon processor 64 GB of DDR3 memory, and two $3.2 \mathrm{~TB}$ NAND flash SSD modules attached over two PCIe gen $3 \mathrm{x} 8$ interfaces. Each Burst Buffer node is attached to a Cray Aries network interconnect over a PCIe gen3 x16 interface. Each Burst Buffer node provides approximately $6.4 \mathrm{~TB}$ of usable capacity and a peak of approximately $5.7 \mathrm{~GB} / \mathrm{sec}$ of sequential read and write bandwidth 13. The maximum write speed of Cori when working properly is twice the speed of Lustre. Also, Burst Buffer is just as fast reading from memory forwards as it is backwards.

The DataWarp software presents to the application a POSIX filesystem interface built on the SSDs. However, unlike a traditional parallel filesystem, this mount is only available to the compute nodes using it, and for limited duration. Therefore, the Burst Buffer has the potential for considerably higher performance than the underlying parallel file system for a variety of reasons, including the underlying storage medium and the high-performance network connection, as well as the possibility to limit metadata load by exposing only the namespace required for the job or workflow.

In previous bandwidth benchmarks, Burst Buffer was shown to outperform the Lustre scratch filesystem. Lustre achieved 
around 700-750 GB/s peak (POSIX File-Per-Process Read/Write) bandwidth and 573/223 GB/s Read/Write performance for MPI-IO shared file. On the other hand, the Burst Buffer system provided approximately $900 \mathrm{~GB} /$ second of peak I/O performance. However, the MPI-IO shared file bandwidth for the Burst Buffer was not faster than Lustre in previous benchmark studies; this is expected to be improved in future versions of the DataWarp software by using more sub-stripes on the underlying Burst Buffer nodes and increased parallelism 4]. Figure 1 illustrates the Cori and Burst Buffer system architecture.

\section{MATERIALS AND METHODS}

The genome assembly application we profiled in this study differs from previous benchmarks in that it reads/writes a large number of rather small files; up to one million files of sizes $100 \mathrm{k}-300 \mathrm{k}$ bytes each. To analyze the performance benefits of Cori and the Burst Buffer over Genepool, we assembled the following genome data sets, all of which were sequenced at JGI:

- Microbes sequenced on PacBio: Meiothermus (315,539 reads), Pedobacter Heparinus (129,154 reads), Escherichia Coli $(225,344$ reads)

- Fungi sequenced on PacBio: Trichoderma Reesei $(382,619$ reads), Phanerochaete Chrysosporium (2,360,808 reads), Scytinostroma sp. KUC9413 (2,198,966 reads)

To get an understanding of Falcon's I/O bandwidth requirements on these data sets, we first ran Falcon on two of the Fungal genome assemblies on Genepool. For both runs the real (wall clock) time was significantly higher than the user and sys time spent in CPU execution within the process. Since real time includes the waiting time for I/O the significantly higher real time shows the processes were I/O bound. In fact the Falcon pipeline consists of several I/O intensive steps, including raw data preprocessing, error-correction of reads, alignment of reads and assembly:

- The first Fungal genome had an expected size of $40 \mathrm{M}$ nucleotides. During the first Falcon step (raw data preprocessing and error-correction of reads) there were 31,815 files of sizes ranging from $320 \mathrm{~KB}$ to $390 \mathrm{~KB}$ written to disk during 63 concurrently running jobs. During the second Falcon step (alignment of reads) there were 2,907 files of sizes ranging from $60 \mathrm{~KB}$ to $80 \mathrm{~KB}$ written to disk during 19 concurrently running jobs. We evaluated the runtimes of all these jobs and found out for the first step the average real time (average over all jobs) was 30s while both user and sys time was $1 \mathrm{~s}$. For the second step the average real time was $5 \mathrm{~s}$, user time $0 \mathrm{~s}$ and sys time $0.2 \mathrm{~s}$.

- The second Fungal genome had an expected size of 33M nucleotides. During the first Falcon step (raw data preprocessing and error-correction of reads) there were 55,195 files of sizes ranging from $220 \mathrm{~KB}$ to $280 \mathrm{~KB}$ written to disk during 83 concurrently running jobs. During the second Falcon step (alignment of reads) there were 657 files of sizes ranging from $50 \mathrm{~KB}$ to $60 \mathrm{~KB}$ written to disk during 9 concurrently running jobs. We evaluated the runtimes of all these jobs and found out for the first step the average real time (average over all jobs) was $25 \mathrm{~s}$ while both user and sys time was $1 \mathrm{~s}$.
For the second step the average real time was $3 \mathrm{~s}$, user time $0 \mathrm{~s}$ and sys time $0.1 \mathrm{~s}$.

For our study, the above data sets were assembled using Falcon, with one compute node, on both Genepool and Cori. Once the assembly was complete, using the bbtools analysis software developed by the JGI [5], the quality of the assembled sequences were evaluated to ensure the native genome integrity is maintained throughout the assembly process on each test case. N50 and L50 are statistics that are widely used to define assembly quality. They are similar to a median of the lengths of the assembled scaffolds. N50 is defined as the length of the shortest scaffold that is at $50 \%$ of the total assembled genome size. N50 is computed by summing the scaffold lengths and ordering them by their length from longest to shortest. L50 is defined as the smallest number of scaffolds whose length sum equals N50 10. Depending on the dataset quality, polymorphism, and repetitiveness of the target genome, massively parallel sequencing technologies can generate draft genomes with N50 statistics ranging from 5kb-200kb. Assemblies with small N50 or high L50 statistics suffer from having many gene models (the foundation of most biological research) with gaps, missing exons, genes split between scaffolds, or missing entirely 9 . For this study, we are mainly concerned with achieving a high quality of genome assemblies and increasing the assembly execution runtime speed. Therefore, our first check was to compare the genome assemblies for our test cases against the published assemblies to ensure consistency between the assemblies produced. All results reported are for high-quality genome assemblies. For example, for Fungal Trichoderma Reesei the assembly statistics for all runs stayed within the ranges of $32-44$ scaffolds, L50 between $4-5$, N50 between $2.9 \mathrm{mb}-4.1 \mathrm{mb}$ and the genome assembly size remained $35 \mathrm{mb}$. For the larger Fungal Scytinostroma sp. KUC9413 the assembly statistics for all runs stayed within the ranges of 307-354 scaffolds, L50 between $31-35$, N50 between $389 \mathrm{~kb}-441 \mathrm{~kb}$ and the genome assembly size remained $46 \mathrm{mb}$.

\section{EVALUATION}

A single test case includes a genome assembly's wall clock runtime on a single node using Genepool local disk (scratch), Genepool GPFS file system, Cori Lustre file system, Cori Lustre file system with Docker, Cori Burst Buffer, and Cori Burst Buffer with Docker. We ran each genome assembly 3 times for each environment and configuration. The main purpose of our evaluation is to compare the genome assembly runtimes on Genepool vs. Cori with the Lustre file system and the Burst Buffer to determine if running on a supercomputer improves the runtimes, as well as the optimal environment for the runs. We compared the effectiveness of the Burst Buffer technology by staging the fragmented sequenced genome data into the Cori's Burst Buffer instead of using the Lustre filesystem. The runtimes were also compared against the Genepool cluster for both the GPFS filesystem as well as the disk that is local to a node (scratch). The bandwidth of the I/O and the run times are recorded using the profiling tool SAR.

There should be an improvement with Burst Buffer because the bandwidth is larger and less time is spent in $\mathrm{I} / \mathrm{O}$. However, if the Burst Buffer does not provide improvements compared to Genepool GPFS file system and Cori Lustre file system executions, that suggests the Cray Burst Buffer 


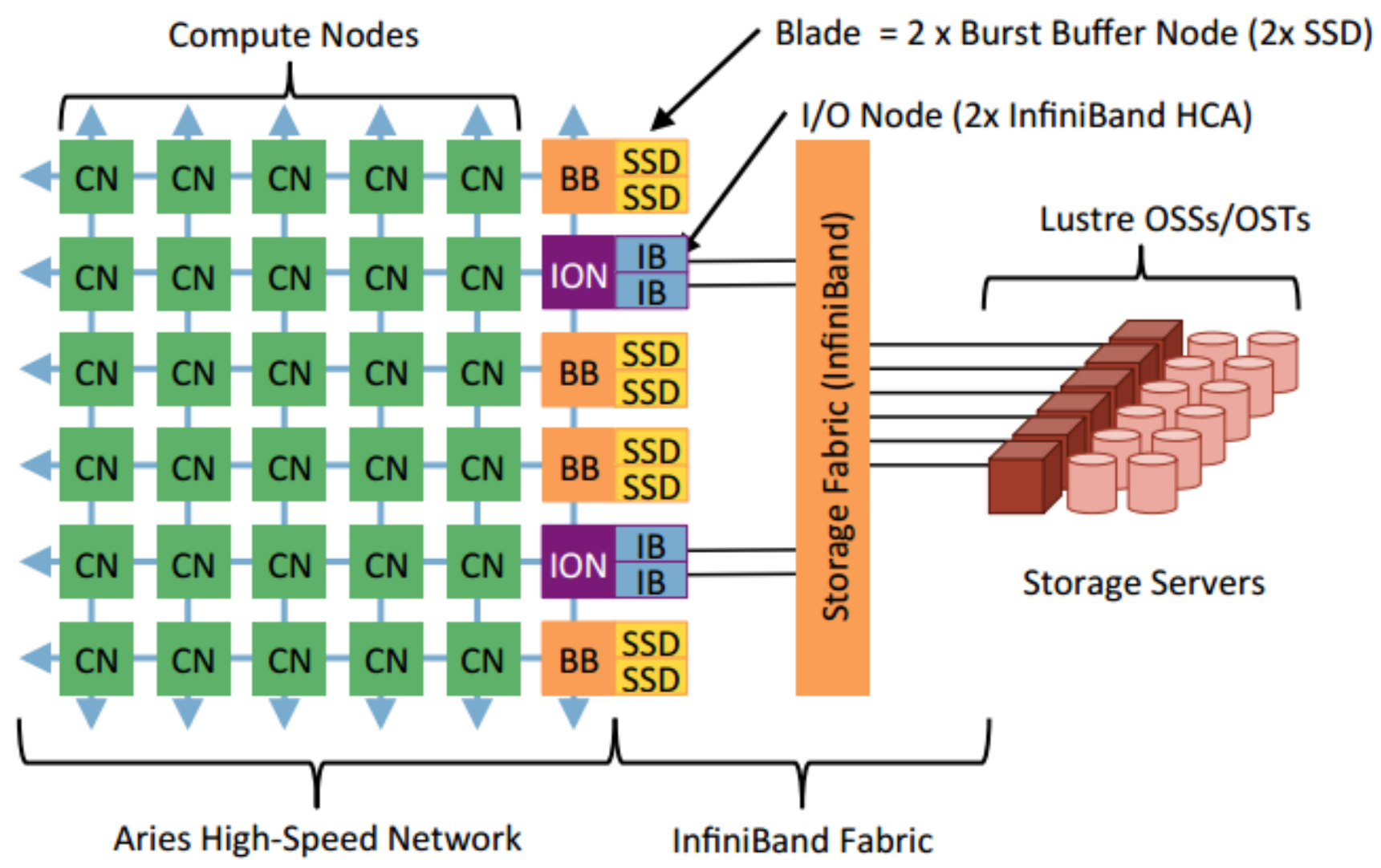

Figure 1: Placement of Burst Buffer nodes in the Cori system architecture 4]. 
may need some tuning and we should inform Cray about the exact tests and results. Cray will pin down what is causing a decrease in performance for the test case and will try to fix any sub-optimal performance issues. Also, if we do not see the performance improvement expected, a next step is to investigate if the metadata servers are causing large delays. In the case of Falcon, which involves reads/writes of many small files, it is possible the metadata servers are the bottleneck. If the performance degrades, we will investigate how the Burst Buffer stores metadata on many small files and provide Cray with information on metadata delays. The tool SAR will indicate if the $\mathrm{I} / \mathrm{O}$ is faster with the Burst Buffer as compared to the Lustre filesystem. In cases where SAR is not available, we could test performance simply by reading and writing a large file in a Burst Buffer interactive session and verify if it is faster than doing the same I/O on Lustre. In the end, if Burst Buffer is performing correctly and scales up to many small files, we expect the genome assembly's runtime to improve.

\subsection{Docker Container for the Falcon Genome Assembly Software}

NERSC has been working to increase flexibility and usability of its high-performance computing systems by enabling Docker-like container technology. Docker containers allow an application to be packaged with its entire software stack - including some portions of the base OS files - as well as defining needed user environment variables and application "entry point." Containers provide an abstract way of deploying portable applications and even automating the execution without requiring detailed tuning or modification to operate them 12 .

To allow comparing the runtimes independent of the time taken for library downloads, we made a Docker container for the Falcon assembler and published the Docker image on Docker Hub 1]. Due to the current configuration of Cori, the python dependencies that are needed to execute Falcon are not pre-installed on cscratch Lustre, but instead have to be loaded into memory before each execution from GPFS (the Genepool file system). This process increases the runtime of Falcon based assemblies. Thus the results may seem like software execution takes longer on Cori compared to Genepool when in reality Cori spends time in the initializing step fetching dependencies, whereas Genepool has them pre-installed. A solution to the problem is to use a Docker image to store Falcon, python, and all its dependencies to ensure the runtime evaluations only consist of the assembly itself and not fetching. This will promote accurate comparisons between different environments. We used Docker for both the Lustre and Burst Buffer (local disk) runs on Cori in order to profile and compare the runtimes of Falcon on the different environments. Docker allowed to ignore external factors across the Lustre and Burst Buffer environments and compare just the assembly execution runtimes.

\section{RESULTS}

The assembly of microbial datasets (Meiothermus, Pedobacter, and Escherichia Coli) and the fungal datasets (Trichoderma Reesei, Phanerochaete Chrysoporium, and Scytinostroma sp. KUC9413) are based on sequencing data produced by PacBio technology. The results in Table 1 show that when running Falcon on the Genepool cluster to assemble the microbial sequences on a single node, the run-times are consistently faster using local scratch (local disk to the node) compared to the higher capacity, off node GPFS file system. This increase in speed is expected on Genepool local scratch, since the bandwidth is higher on on-node memory as the data has a shorter distance to travel. The fungal assembly runtimes (shown in Table 1) on Genepool further meets our expectations, as they are consistently faster on local scratch compared to GPFS.

The results in Table 2 show that when running Falcon on Cori Lustre and Cori Lustre with Docker to assemble the microbial sequences on a single node, the run-times are consistently faster without using Docker compared to using Lustre with Docker. Theoretically, Cori with Lustre and Docker should outperform Cori with Lustre only as Falcon, Python, and all its library dependencies are pre-loaded onto a Docker image that acts as a virtual run-time environment to execute the assemblies. Meaning the time spent fetching the dependencies is eliminated. This result suggests that there may have been a bug at the time of the benchmark run in the Docker adaptation or configuration on Cori. The fungal executions (shown in Table 2) on Cori Lustre further defies our expectations, as they are consistently faster on Lustre compared to Lustre with Docker. The Docker platform on Cori is currently under investigation.

Using Docker also did not improve the runtime on Cori when using the Burst Buffer. The results in Table 3 show that when running Falcon on the Burst Buffer, the run-times are consistently faster using Burst Buffer without Docker compared to using Burst Buffer with Docker. This holds for all assemblies of microbial or fungal sequences on a single node. Theoretically, Cori on Burst Buffer with Docker should outperform Cori with Burst Buffer only as Falcon, Python, and all its library dependencies are pre-loaded onto a Docker image that acts as a virtual run-time environment to execute the assemblies. Meaning the time spent fetching dependencies is eliminated. This result suggests that there may have been a bug in the Docker configuration on Cori.

Figure 2 shows the average runtimes of microbial genomes (Meiothermus, Pedobacter, Escherichia Coli) and fungal genomes (Trichoderma Reesei, Scytinostroma) assemblies using Falcon on Genepool and the Cori XC40 supercomputer on a single compute node. As mentioned above, the assemblies were executed under 6 different environments: 2 on Genepool (local disk scratch on the node and GPFS projectb) and 4 on Cori (Lustre cscratch and Burst Buffer with and without Docker). We ran each test case 3 times on each configuration and environment and computed the average run-time over the 3 runs. The results show that Genepool is working as expected as the assemblies using local disk scratch are consistently faster than Genepool using GPFS (projectb). Cori with Lustre (cscratch) is consistently the fastest overall. The local Burst Buffer was not more efficient than Lustre cscratch on Cori. The results suggests that misconfigurations are likely still impeding Burst Buffer's performance; this is expected since the Burst Buffer technology is still undergoing testing and evaluation.

\section{DISCUSSION}

As Figure 2 shows, the runtimes on Cori with the Lustre file system (cscratch) were the fastest overall. However, there are still issues that need to be resolved before the JGI can move its assembly runs (and potentially other pipelines) to the Cori supercomputer. One significant issue was that 


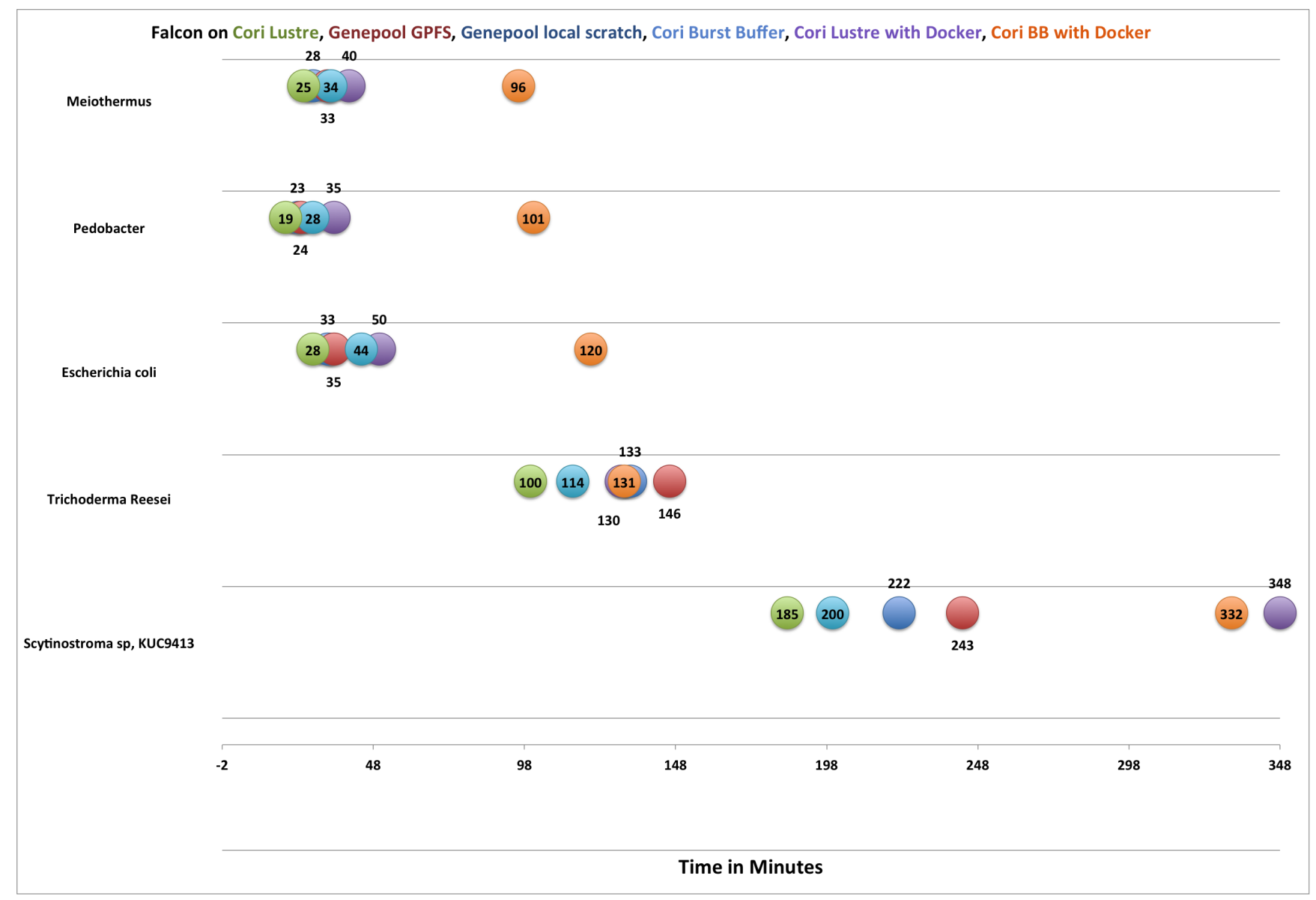

Figure 2: Runtimes of Falcon dot graph. Each dot is the average run-time over the 3 tests for a particular genome and computation environment. 
Table 1: Genepool assembly run-times in minutes for local (scratch) disk and GPFS (projectb) file system

\begin{tabular}{l|ll}
\hline & Genepool local scratch & Genepool GPFS file system \\
\hline Meiothermus & $29,29,28$ & $36,30,32$ \\
Pedobacter Heparinus & $23,23,23$ & $25,24,23$ \\
Escherichia Coli Str & $33,33,33$ & $35,35,34$ \\
Trichoderma Reesei & $133,145,143$ & $154,143,140$ \\
Phanerochaete Chrysosporium & $135,135,132$ & $143,140,100$ \\
Scytinostroma sp. KUC9413 & $222,218,238$ & $249,240,241$ \\
\hline
\end{tabular}

Table 2: Cori assembly run-times in minutes for the Lustre (cscratch) file system

\begin{tabular}{l|ll}
\hline & Cori Lustre cscratch & Cori Lustre cscratch with Docker \\
\hline Meiothermus & $25,27,24$ & $40,39,42$ \\
Pedobacter Heparinus & $19,20,19$ & $34,35,37$ \\
Escherichia Coli Str & $28,28,27$ & $50,50,51$ \\
Trichoderma Reesei & $105,98,98$ & $128,131,131$ \\
Phanerochaete Chrysosporium & $>12 \mathrm{hrs}$ & $>12 \mathrm{hrs}$ \\
Scytinostroma sp. KUC9413 & $193,181,180$ & $342,348,354$ \\
\hline
\end{tabular}

Table 3: Cori assembly run-times in minutes for the Burst Buffer (local disk)

\begin{tabular}{l|ll}
\hline & Cori Burst Buffer & Cori Burst Buffer with Docker \\
\hline Meiothermus & $26,51,24$ & $97,95,95$ \\
Pedobacter Heparinus & $20,35,30$ & $101,100,101$ \\
Escherichia Coli Str & $29,55,50$ & $120,117,122$ \\
Trichoderma Reesei & $112,114,115$ & $132,130,131$ \\
Phanerochaete Chrysosporium & $>12 \mathrm{hrs}$ & $>12 \mathrm{hrs}$ \\
Scytinostroma sp. KUC9413 & $202,199,198$ & $326,340,329$ \\
\hline
\end{tabular}

the Phanerochaete Chrysosporium fungal genome took over 12 hours to assemble on Cori, whereas on Genepool with local scratch it took 135 minutes of wall clock run-time. This should not be possible and points to an edge case.

Docker did not result in a runtime improvement on Cori , so the Docker implementation on Cori (Shifter) is currently under investigation. We will try compiling the Docker image on Cori as this will use Cray-specific libraries that may improve the runtimes. The Genepool results were much faster on local disk (scratch) as opposed to GPFS, which was also an expected result. We expected the runtimes on Cori with Burst Buffer to be faster than the Cori Lustre executions, based on the hardware specifications of the Burst Buffer. The Burst Buffer on Cori did not improve runtimes significantly. Although we were hopeful to see significant improvement with the Burst Buffer, especially when using Docker, the results do not come as a total surprise. Cori with the Burst Buffer is still in the testing phase and it has not been tuned yet for projects like genome assembly that write a large number of small files (e.g. half a million files of sizes 200K bytes each). Rather, Burst Buffer previously showed bandwidth and runtime improvements when writing a small number of large files. The research showed that Cori with Lustre results in improved runtimes compared to the Genepool cluster, but fine tuning of the Burst Buffer (local storage on Cori) is still needed for our application. We will report the test cases and data to Cray as they continue to service Cori, as a resource to improve runtimes and help tune the Burst Buffer for future use.

We also tried using more than one data node on Burst Buffer, as well as more than one compute node on Cori, to see how much runtime and $\mathrm{I} / \mathrm{O}$ bandwidth increase is possible per additional node; using additional Burst Buffer data nodes or Cori compute nodes did not result in any runtime improvement. Throughout this research project, we experienced several bugs that have completely crashed the Burst Buffer altogether. One bug that often crashed the system is requesting 31 nodes in a SLURM sbatch script.

\section{FUTURE WORK}

Because of the runtime improvement that using Burst Buffer on Cori was expected to show, the observation of both Genepool and Cori with Lustre outperforming Burst Buffer leads to the conclusion that some fine tuning is necessary for the current configuration of the Burst Buffer. We will submit its test cases and data to Cray as a resource to help tune or pin down bugs involving the Burst Buffer. Until that time, we plan to run more bioinformatics pipelines on Cori with the Lustre file system. Additionally, we would like to explore assembling plant genomes, since plants have a large genome size and would require more I/O, and thus will serve as a better stress tester of the Burst Buffer capabilities. The Docker image for Falcon is currently being re-built on the Cray using Cray-specific libraries and mkl for numpy; the re-build with Cray-specific libraries might improve the runtime of the Docker image on Cray. Finally, iobuf is a Cray library for caching/buffering that has just been built on Cori for Burst Buffer and it remains to be seen if iobuf will improve the runtimes.

\section{ACKNOWLEDGMENTS}


This work was supported in part by the U.S. Department of Energy, Office of Science, Office of Workforce Development for Teachers and Scientists (WDTS) under the Science Undergraduate Laboratory Internship (SULI) program. The authors would like to give special thanks to Laleh Cote, Nakeiah Harrell, Kjiersten Fagnan, Alicia Clum, the National Energy Research Scientific Computing Center (NERSC), The Joint Genome Institute, and The University of Alabama at Birmingham.

\section{REFERENCES}

[1] B. Andreopoulos. [accessed July 1, 2016]. https: //hub.docker.com/r/billandreo/falcon_ctr5/

[2] A. Bankevich, S. Nurk, D. Antipov, A.A. Gurevich, M. Dvorkin, A.S. Kulikov, V.M. Lesin, S.I. Nikolenko, S. Pham, A.D. Prjibelski, A.V. Pyshkin, A.V. Sirotkin, N. Vyahhi, G. Tesler, M.A. Alekseyev, P.A. Pevzner. SPAdes: a new genome assembly algorithm and its applications to single-cell sequencing, J Comput Biol 19(5):455-77, 2012. doi: 10.1089/cmb.2012.0021.

[3] D.A. Benson. GenBank. Nucleic Acids Research, 38 (Database issue) Nucleic Acids Research, 38 (Database issue) D46-D51, 2010.

[4] W. Bhimji, D. Bard, M. Romanus, D. Paul, A. Ovsyannikov, B. Friesen, M. Bryson, J. Correa, G.K. Lockwood, V. Tsulaia, S. Byna, S. Farrell, D. Gursoy,C. Daley,V. Beckner, B. Van Straalen, N.J. Wright, K. Antypas, Prabhat. Accelerating Science with the NERSC Burst Buffer Early User Program, Cray User Group, May 11, 2016.

[5] B. Bushnell. [accessed July 1, 2016]. https://sourceforge.net/projects/bbtools/

[6] J. Butler, I. MacCallum, M. Kleber, I.A. Shlyakhter, M.K. Belmonte, E.S. Lander, C. Nusbaum, D.B. Jaffe. ALLPATHS: de novo assembly of whole-genome shotgun microreads, Genome Research 18: 810âĂŞ20, 2008.

[7] J. Chin. https://github.com/PacificBiosciences/FALCON/

[8] Cray. DataWarp User Guide, 2016. Available Online: http://docs.cray.com/books/S-2558-5204/ S-2558-5204.pdf

[9] A.C. English. Mind the Gap: Upgrading Genomes with Pacific Biosciences RS Long-Read Sequencing Technology, PLoS ONE 7(11), 2012: e47768. doi: 10.1371/journal.pone.0047768

[10] D. Kleftogiannis, P. Kalnis, V. B. Bajic. Comparing Memory-Efficient Genome Assemblers on Stand-Alone and Cloud Infrastructures, PLoS ONE 8(9): e75505, 2013. doi:10.1371/journal.pone.0075505.

[11] N. Nagarajan and M. Pop. Sequence assembly demystified. Nature Reviews Genetics 14, 157-167, March 2013. doi:10.1038/nrg3367

[12] NERSC. Shifter: Bringing Linux containers to HPC. [accessed April 20, 2016]. http://www.nersc.gov/ research-and-development/user-defined-images/

[13] NERSC. Burst Buffer Architecture and Software Roadmap. [accessed April 22, 2016]. http: //www.nersc.gov/users/computational-systems/ cori/burst-buffer/burst-buffer/
[14] M. Pop. Genome assembly reborn: recent computational challenges. Briefings in Bioinformatics, 10: 354-366, 2009.

[15] D.R. Zerbino and E. Birney. Velvet: Algorithms for de novo short read assembly using de Bruijn graphs. Genome Research 18(5):821-829, 2008. doi:10.1101/gr.074492.107. 